\title{
Martial Arts Training Attenuates Arterial Stiffness in Middle Aged Adults
}

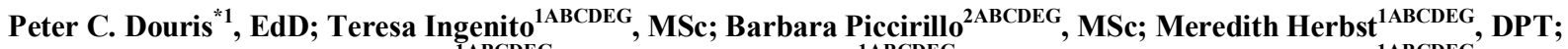
John Petrizzo $^{1 \mathrm{ABCDEG}}$, DPT; Vincen Cherian ${ }^{1 \mathrm{ABCDEG}}$, DPT; Christopher McCutchan ${ }^{1 \mathrm{ABCDEG}}$, DPT; Caitlin Burke $^{1 \mathrm{ABCDEG}}$, DPT; George Stamatinos ${ }^{1 \mathrm{BBCDEG}}$, DPT; Min-Kyung Jung ${ }^{3 \mathrm{CDG}}$, PhD

\author{
Authors' Affiliation: \\ 1. Department of Physical Therapy, New \\ York Institute of Technology, USA \\ 2. Department of Physician Assistant \\ Studies, New York Institute of \\ Technology, USA \\ 3. New York York Institute of Technology \\ College of Osteopathic Medicine, USA

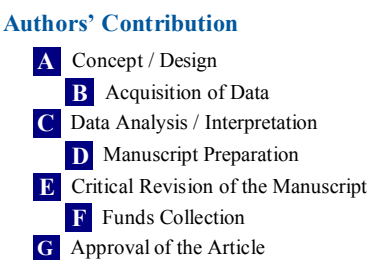 \\ * Corresponding Author; \\ Address: Department of Physical Therapy \\ New York Institute of Technology \\ Old Westbury, NY 11568-8000, USA \\ E-mail: pdouris@nyit.edu
}

Received: Dec 07, 2012

Accepted: $\operatorname{Mar} 24,2013$

Available Online: $\operatorname{Apr} 21,2013$

\begin{abstract}
Purpose: Arterial stiffness increases with age and is related to an increased risk of coronary artery disease. Poor trunk flexibility has been shown to be associated with arterial stiffness in middle-aged subjects. The purpose of our research study was to measure arterial stiffness and flexibility in healthy middle-aged martial artists compared to age and gender matched healthy sedentary controls.

Methods: Ten martial artists $(54.0 \pm 2.0$ years $)$, who practice Soo Bahk Do (SBD), a Korean martial art, and ten sedentary subjects (54.7 \pm 1.8 years) for a total of twenty subjects took part in this cross-sectional study. Arterial stiffness was assessed in all subjects using pulse wave velocity (PWV), a recognized index of arterial stiffness. Flexibility of the trunk and hamstring were also measured. The independent variables were the martial artists and matched sedentary controls. The dependent variables were PWV and flexibility.

Results: There were significant differences, between the SBD practitioners and sedentary controls, in PWV $(P=0.004)$, in trunk flexibility $(P=0.002)$, and in hamstring length $(P=0.003)$.

Conclusion: The middle-aged martial artists were more flexible in their trunk and hamstrings and had less arterial stiffness compared to the healthy sedentary controls. The flexibility component of martial art training or flexibility exercises in general may be considered as a possible intervention to reduce the effects of aging on arterial stiffness.
\end{abstract}

Key Words: Compliance; Flexibility; Karate; Sedentary; Elasticity

\section{INTRODUCTION}

A rterial stiffness or lower arterial elasticity has been shown to have a direct association with aging and is a hallmark indicator of cardiovascular health as well as cardiorespiratory fitness ${ }^{[1,2]}$. The stability, resilience and compliance of the vascular walls are dependent on the relative contribution of the two prominent scaffolding proteins: collagen and elastin ${ }^{[2,3,4]}$. On a structural basis, arterial stiffness occurs when the elastic fibers within the arterial wall begin to fray due to mechanical stress. Recent evidence strongly supports the hypothesis that arterial stiffness is a risk factor associated with atherosclerosis, coronary artery disease; diabetes, stroke, and end stage renal disease ${ }^{[1,2,5,6]}$. Accordingly, Sutton-Tyrrell et al ${ }^{[7]}$ have provided evidence that decreased arterial compliance in an otherwise healthy middle-aged population is an independent marker of cardiovascular mortality.

The two main treatment strategies to maintain or improve arterial compliance are pharmacologic management, and exercise ${ }^{[2,8]}$. There is growing research to support the use of aerobic training as an effective intervention for management of the agerelated decrease of arterial compliance ${ }^{[9-11]}$. Similarly, research has also shown that resistance training does not negatively affect arterial stiffness in middle-aged adults ${ }^{[12-14]}$. Do the age-related changes that decrease the flexibility of the connective tissues within muscle and tendon similarly affect the connective tissues 
within the vascular walls? Flexibility in general starts to decline in middle age ${ }^{[15,16]}$. According to the American College Sports Medicine, ${ }^{[15]}$ flexibility is a chief component of physical health and fitness especially starting in middle age. However, there remains a paucity of information regarding the relationship between arterial stiffness and flexibility.

Cortez-Cooper et al ${ }^{[12]}$ compared 13 weeks of strength training, strength training plus aerobic exercise, and stretching as the control group, in middle and older sedentary subjects. They found that arterial and actually improved in the stretching group. The practice of Tai Chi, an ancient Chinese martial art, in older adults (mean age 73.7 \pm 4.5 ) that includes flexibility exercises has been shown to decrease arterial stiffness [17]. Yamamoto et al ${ }^{[4]}$ investigated the relationship between arterial stiffness and flexibility in young, middle and older aged subjects. They further divided subjects into two groups, poor and high flexibility, based on the results of a sit-and-reach test. They discovered that there was a significant difference in arterial stiffness between the poor and high flexibility groups starting in the middle and older aged subjects. They concluded that greater flexibility was associated with decreased age-related arterial stiffness independent of cardiovascular fitness and muscle strength. However, they did not specify what type of exercise their subjects performed in order to maintain their flexibility, nor did they examine if subjects that were more flexible actually stretched to maintain their flexibility. This current gap in the knowledge illustrates the need for additional research to examine the role of flexibility training on arterial stiffness specifically within a middle-aged population who regularly practice flexibility exercises. Flexibility exercises are the foundation of martial art training.

The purpose of this study was to examine the difference between arterial stiffness and flexibility in middle-aged martial artists compared to sedentary controls. We hypothesized that middle-aged martial art practitioners will possess decreased arterial stiffness and greater trunk and hamstring flexibility when compared to age and gender matched sedentary controls.

\section{METHODS AND SUBJECTS}

\section{Subjects:}

The study consisted of 20 middle-aged subjects divided into two stratified groups, one group of 10 martial artists $(54.0 \pm 2.0$ years), who practice Soo Bahk Do, a Korean martial art, and another group of 10 sedentary control subjects $(54.7 \pm 1.8$ years). The ages for both groups ranged from 40-64 years old. This study was a matched design; subjects were matched by age (within 2 years) and gender. There were 7 men and 3 women in each of the two groups. The martial artists were volunteers from the United States Soo Bahk Do Federation. Soo Bahk Do (SBD) is traditional Korean martial art. SBD training has been shown to increase the overall physical fitness and health of middle-aged practitioners $[18,19]$. The sedentary subjects were randomly selected volunteers within the New York Institute of Technology (NYIT) community. Before starting, all subjects gave written informed consent. Subjects completed a medical history and health questionnaire to screen for inclusion, exclusion criteria and to confirm a sedentary lifestyle in the control subjects $[18,19]$. Inclusion criteria were martial art practitioners who have been practicing for at least seven years at a minimum of twice per week with a rank of black belt. The exercise regimen for the martial art practitioners was primarily participation in SBD training. SBD is a traditional Korean martial art similar to karate. A typical SBD class is one hour in length. A typical class usually includes a 15-minute warm up of flexibility exercises and calisthenics. The core of the class may include 15 minutes of basic kicking, blocking and punching drills, 15 minutes of hyung (set forms in pre-established sequences of defensive and offensive movements), 10 minutes of sparring against an opponent and self defense techniques. The class typically ends with a 5-minute cool down of calisthenics and flexibility exercises ${ }^{[19]}$. The practice of SBD as with most martial arts includes a great deal of stretching exercises and movements requiring total body flexibility and that was the rationale for choosing SBD practitioners as subjects to investigate for our study. Flexibility exercises are the cornerstone of martial art training. The martial art practitioners in the present study were all regularly practicing black belts 
with an average experience of approximately twenty years in SBD. Sedentary subjects were defined as those who did not participate in any formal program of physical exercise including any form of flexibility training other than activities that are functionally and occupationally related. Exclusion criteria for all subjects consisted of diabetes mellitus, cardiovascular disease, taking medications for hypertension or hypercholesterolemia, current cigarette smokers and any major musculoskeletal injuries or pathologies of the spine and lower extremities within the past twelve months. The institutional review board at NYIT approved this research.

\section{Study design:}

This was a cross-sectional design utilizing middle-aged martial artists and sedentary controls. Each subject made only one visit for testing. Testing was performed in the morning hours, subjects were asked to eat a light breakfast, not consume caffeine, or engage in strenuous physical activity 24 hours before testing. Anthropometric measures of height and weight were measured in standing. Subjects were then asked to rest in the supine position for 5 minutes before hemodynamic assessments. In the supine postion, with the subject's trunk inclined at a 30-degree angle, blood pressure, heart rate and pulse wave velocity (PWV) were measured. Trunk flexibility was measured following the measurement of the hemodynamic parameters utilizing the sit-and-reach test and hamstring flexibility was measured with a goniometer.

\section{Anthropometric measures:}

Height and weight were measured wearing lightweight clothing and bare feet. Height was measured with a standard fixed stadiometer and weight was measured on a calibrated standard beam balance scale. Body mass index (BMI) was calculated as subject's weight divided by their height in meters squared.

\section{Hemodynamic measures:}

Brachial blood pressure (BP) was measured on the subject's left arm with the subject's trunk inclined at a 30-degree angle with a mercury sphygmomanometer. The methods have been previously described and approved by the American Heart Association ${ }^{[20]}$.
Arterial stiffness measurements were measured first followed by flexibility measurements. Pulse Wave Velocity (PWV) is the current gold standard of measuring arterial stiffness ${ }^{[21]}$. The Vicoder System (Skidmore Medical, Bristol, UK) was used to measure the subjects' arterial stiffness. The Vicorder instrument is a medical device intended for the investigation of vascular disorders. One $30 \mathrm{~mm}$ cuff was placed around the subject's neck with the pressure pad over the right carotid area and secured with Velcro fixation. A second $100 \mathrm{~mm}$ wide cuff was placed around the subject's upper right thigh with the pressure pad secured over the area of the right femoral artery. The distance between the suprasternal notch and the superior border of the thigh cuff was measured in centimeters and entered into the system. The cuffs were then inflated using the Vicoder system to $60 \mathrm{mmHg}$ and the high quality waveforms were recorded simultaneously for 3 seconds using a volume displacement method. The foot-to-foot transit time was calculated using an in-built, crosscorrelational algorithm centered around the peak of the second derivative of pressure ${ }^{[21]}$. The PWV was recorded in meters per second. The Vicorder also recorded heart rate in beats per minute. The validity and reliability of the Vicorder has been established by Hickson et al ${ }^{[21]}$.

\section{Flexibility measures:}

A standard sit-and- reach box was used to measure the flexibility or range of motion (ROM) of the trunk in centimeters ${ }^{[22,23]}$. Subjects did not perform any type of specific warm-up before performing the sit-and-reach test. Subjects were placed in a seated position with their feet placed under the lip of the box. They were then instructed to keep their legs straight throughout each trial as well as to begin each trial with their back fully erect. Subjects were asked to overlap their hands (as if diving) and extend the arms as far forward as possible. The subject would slowly reach along the top of the test box towards the feet until forward motion stopped. Bouncing to end ROM was not allowed. The subject then returned to the starting position and repeated the cycle two more times ${ }^{[22,23]}$. The best measurement of the three trials was used for statistical analysis. A similar protocol was utilized by Yamamoto et $\mathrm{al}^{[4]}$. Following the conclusion of the sit-and-reach 
test, subjects were assessed for passive hamstring length. According to Baltaci et al ${ }^{[23]}$, the passive straight leg raise has both a high sensitivity and specificity in its ability to assess passive extensibility of the hamstring muscles. The procedure used to perform the passive straight leg raise was as follows: the axis of the goniometer was aligned with the subject's greater trochanter. The tester positioned the stationary arm in line with the trunk, placed the moving arm in line with the lateral femoral condyle and with the knee held straight, the subject's leg was moved passively into hip flexion until the initial feeling of tightness ${ }^{[23]}$. The tester then read the goniometer in degrees of motion. Three trials were performed on each leg and the highest score of each leg was averaged between the two legs. That score in degrees was utilized for subsequent statistical analysis.

\section{Statistical Analyses:}

This study was a cross sectional study utilizing a matched pairs design in order to investigate the difference between martial art training and a sedentary lifestyle on flexibility and arterial stiffness. Statistical analyses were performed utilizing SPSS for Windows (version 20.0, Chicago, Ill.). The characteristics of age, height, weight, BMI, heart rate, systolic and diastolic blood pressure were compared between the groups by utilizing paired t-tests that were adjusted with the bonferroni correction to control for the family-wise error rate and reduce the potential for Type 1 errors ${ }^{[24,}$ ${ }^{25]}$. The independent variables were the matched martial artists (SBD) and sedentary individuals (SED). The dependent variables were PWV, hamstring length, and trunk flexibility (sit-and-reach). Significant differences between the groups for the dependent variables were assessed by a multivariate analysis of variance (MANOVA). The significance level was set at $P \leq 0.05$. Comparisons were performed with univariate analysis that was adjusted with the bonferroni correction if a statistical significance were found in the multivariate test.

\section{RESULTS}

The physiological characteristics of the subjects are presented in Table 1. The two groups did not differ significantly in terms of their physiological characteristics. The MANOVA was statistically significant $(P=0.001)$. This allowed us to perform comparisons utilizing univariate analysis between the dependent variables. The results of the pairwise comparisons and the means $( \pm$ SEM) of each group are presented in Table 2. The SBD practitioners had a significantly $\left(P=0.004, \eta^{2}=0.38\right)$ lower $\mathrm{PWV}$ as compared to their sedentary counterparts. Between group differences were also seen when comparing trunk flexibility $\left(P=0.002, \eta^{2}=0.43\right)$ and hamstring flexibility $\left(P=0.003, \eta^{2}=0.41\right)$.

\section{DISCUSSION}

This study has provided evidence that practicing martial arts, specifically SBD, may be an effective intervention attenuating the effects of normal aging on arterial stiffness in middle-aged persons. The effects of

Table 1: Physiological characteristic of subjects

\begin{tabular}{|lcc|}
\hline Parameter & $\begin{array}{c}\text { Soo Bahk Do }(\mathbf{n = 1 0}) \\
\text { Mean (SEM) }\end{array}$ & $\begin{array}{c}\text { Sedentary (n=10) } \\
\text { Mean (SEM) }\end{array}$ \\
\hline Age (yr) & $54.00(2.02)$ & $54.70(1.75)$ \\
Height (cm) & $171.39(2.60)$ & $174.30(2.42)$ \\
Weight (Kg) & $75.64(4.71)$ & $89.79(0.58)$ \\
Body Mass Index (kg. m-2) & $25.28(1.01)$ & $29.28(1.01)$ \\
Heart Rate (b/min) & $59.80(2.53)$ & $63.40(3.77)$ \\
Systolic Blood Pressure (mmHg) & $111.90(3.80)$ & $122.90(3.75)$ \\
Diastolic Blood Pressure (mmHg) & $69.40(2.15)$ & $74.20(2.49)$
\end{tabular}


Table 2: Comparison of results between the soo bahk do and sedentary subjects

\begin{tabular}{|c|c|c|c|c|}
\hline Parameter & $\begin{array}{c}\text { Soo Bahk Do }(n=10) \\
\text { Mean (SEM) }\end{array}$ & $\begin{array}{c}\text { Sedentary }(\mathrm{n}=10) \\
\text { Mean }(\mathrm{SEM})\end{array}$ & $\begin{array}{c}\text { Observed } \\
\text { Power }\end{array}$ & $P$. value \\
\hline Pulse Wave Velocity $(\mathrm{m} / \mathrm{s})$ & $7.60(0.85)$ & $18.15(3.07)$ & 0.88 & 0.004 \\
\hline Trunk (cm) & $48.05(3.18)$ & $30.65(3.49)$ & 0.94 & 0.002 \\
\hline Hamstring (d) & $78.10(2.64)$ & $65.20(2.56)$ & 0.91 & 0.003 \\
\hline
\end{tabular}

normal aging have been shown to be directly related with increasing arterial stiffness starting in middleaged persons ${ }^{[2,4]}$. According to Vlachopoulos et al ${ }^{[26]}$ arterial stiffness is a strong predictor of future cardiovascular events and all cause mortality.

The SBD subjects in this study had significantly less arterial stiffness and greater trunk and hamstring flexibility than the sedentary subjects. The mean PWV was $7.60 \pm 0.85 \mathrm{~m} / \mathrm{s}$ with a mean trunk ROM of 48.05 $\pm 3.18 \mathrm{~cm}$ as determined by the sit-and-reach test and mean hamstring length of $78.10 \pm 2.64$ degrees compared to the sedentary subjects' mean PWV of $18.15 \pm 3.07 \mathrm{~m} / \mathrm{s}$, trunk ROM of $30.65 \pm 3.49 \mathrm{~cm}$ and hamstring ROM of $65.20 \pm 2.56$ degrees. Seven of the ten SBD subjects had PWV values below their agepredicted norms while all ten of the sedentary subjects exceeded their age-predicted norms for healthy PWV ${ }^{[27]}$. The SBD subjects on average were in the $80^{\text {th }}$ percentile for trunk ROM while the sedentary subjects were averaged in the $30^{\text {th }}$ percentile ${ }^{[22]}$. Our results for trunk ROM compare favorably to the middle-age subjects in the Yamamoto et al ${ }^{[4]}$ study. Trunk ROM for their high flexibility and poor flexibility groups was $46.0 \mathrm{~cm}$ and $31 \mathrm{~cm}$, respectively. We also evaluated hamstring length as an additional measure for flexibility and the groups were statistically different in that measurement as well.

To our knowledge, our research is the first study to examine arterial stiffness and flexibility in middle-aged adults who regularly performed flexibility exercise as part of their exercise routine, specifically SBD martial art training in comparison to sedentary subjects matched for sex and age. Our results compare favorably to $\mathrm{Lu}$ et al ${ }^{[17]}$, who concluded that older subjects who practice Tai Chi regularly have better arterial compliance than healthy control subjects. Their cross sectional design was similar to ours, however we investigated a middle aged population, who according to the literature is when arterial stiffness becomes a cardiovascular risk factor ${ }^{[1,2,6,7]}$. Our results also compare favorably to Cortez-Cooper et al ${ }^{[12]}$, who discovered that flexibility exercises in previously sedentary subjects decreased arterial stiffness by $23 \%$ over a 13 -week training period. The SBD subjects in our study have been stretching as part of their SBD regime on average for over twenty years. The key finding in Yamamoto et al ${ }^{[4]}$ cross sectional study which included 104 middle aged subjects was that there was a $30 \%$ significant difference in arterial stiffness between the high and poor flexibility groups. There was a $140 \%$ difference in our study. This discrepancy may be due to that fact that we had fewer subjects and our subjects who demonstrated poor trunk flexibility were sedentary. Yamamoto et al ${ }^{[4]}$ did not differentiate whether the poor flexibility subjects were physically active or sedentary. They also did not specify what type of exercise their subjects performed in order to maintain their flexibility, nor did they examine if subjects that were more flexible actually stretched to maintain their flexibility. They concluded that flexibility might be a predictor of arterial stiffening, independent of other components of fitness, cardiorespiratory fitness and muscle strength ${ }^{[4]}$.

Yamamoto et al ${ }^{[4]}$ proposed two physiological mechanisms to explain the relationship between flexibility and arterial stiffness. The first one is that structures that compose the connective tissues in muscle, tendons, ligaments and arteries are structurally similar. The stability and compliance of these tissues are dependent on the contribution of two scaffolding proteins: elastin and collagen ${ }^{[2]}$. Collagen bears loads in the circumferential direction, whereas elastin offers longitudinal as well as circumferential support ${ }^{[3]}$. Normal aging results in an overproduction of collagen and diminished quantities of elastin resulting in increasing arterial stiffness ${ }^{[2]}$. Morphological changes 
associated with aging result in decreased compliance and flexibility of the connective tissue found within the musculotendinous unit $^{[28]}$. Therefore, age-related changes in arterial stiffness may correspond to agerelated changes in musculotendinous flexibility ${ }^{[4]}$. Maintaining overall flexibility of the musculotendinous units and joint structures may also contribute to maintaining the compliance of the vascular system. The connective tissue of the musculotendinous units and vascular system may respond to the mechanical stress of stretching in a similar manner. The second physiological mechanism proposed by Yamamoto et al [4] is that repetitive sympathetic nervous system stimulation induced by habitual stretching exercises may reduce vascular tone, which in turn may reduce arterial stiffness. The SBD subjects did present with lower but not statistically lower systolic blood pressure and resting heart rates, which may reflect decreased sympathetic tone. The systolic blood pressures of both groups were within normal ranges ${ }^{[20]}$.

The research provides strong evidence of the relationship between decreased arterial stiffness and physical activity ${ }^{[5,9,29]}$. Sedentary middle-aged adults are at a greater risk for cardiovascular complications secondary to decreased arterial compliance without any other symptoms of cardiovascular disease ${ }^{[1,2,6,7]}$. The results of this investigation indicate that even without a history of smoking, diabetes mellitus, high blood pressure, or high cholesterol, a sedentary lifestyle can result in decreased arterial compliance and ultimately increase the risk for cardiac complications. According to Sutton-Tyrell et al ${ }^{[7]}$, asymptomatic individuals with increased arterial stiffness will eventually progress to hypertension.

The limitations of this present study are inherent in the use of a cross-sectional design. The major threat to utilizing a cross sectional design is selection bias and the difficulty in establishing a cause and effect relationship ${ }^{[25]}$. While flexibility is a cornerstone of martial art training, there are also strength and cardiovascular components ${ }^{[18]}$. Lu et al ${ }^{[17]}$ concluded that older Tai Chi subjects besides displaying greater arterial compliance also demonstrated greater muscle strength than there healthy controls. Douris et al ${ }^{[18]}$ also has previously established that SBD practitioners displayed greater lower extremity strength when compared to sedentary controls. However, Yamamoto et $\mathrm{al}^{[4]} \mathrm{did}$ conclude that flexibility may be a predictor of arterial stiffness in middle age and older subjects independent of the other components of fitness. Future studies should investigate different exercise modalities such as yoga or possibly static stretching as a standalone intervention without the potential confounding variables of cardiovascular or strength training. Future research in this area should also examine whether or not flexibility exercise can be used to reverse or modify arterial stiffness in patients who already display decreased arterial compliance. Clinical significance of our study may be that the sedentary lifestyle of middleaged individuals may predispose them to future cardiovascular events secondary to decreased arterial compliance independent of a lack of current cardiovascular risk factors. In addition, our results indicate that flexibility training should be considered as a benefit and integral part of a comprehensive exercise program to modify a patient's risk for age related decreases in arterial compliance and decrease the risk of cardiovascular disease.

\section{CONCLUSION}

Millions of individuals worldwide practice martial arts and it may be considered one of the oldest forms of exercise known to man. The conditioning practices of the martial arts have been passed down from generation to generation for thousands of years and it is only recently that scientific investigation has begun to validate its health and exercise benefits. In conclusion, we were able to demonstrate that the long-term practice of martial art training may be associated with an attenuation of the age related effects on arterial stiffness in middle-aged SBD practitioners.

\section{ACKNOWLEDGMENTS}

We gratefully acknowledge the New York Institute of Technology Institutional Review Board for providing us the permission to perform the study and all the subjects that participated.

Conflict of interests: None 


\section{REFERENCES}

[1] Kingwell BA, Gatzka, CD. Arterial stiffness and prediction of cardiovascular risk. J Hypertens 2002;20:2337-40.

[2] Zieman SJ, Melennovsky V, Kass DA. Mechanism, pathophysiology, and therapy of arterial stiffness. Arterioscler Thromb Vasc Biol 2005;25:932-43.

[3] Silver FH, Horvath I, Foran DJ. Visoelasticity of the vessel wall: the role of collagen and elastic fibers. Crit Rev Biomed Eng 2001; 29: 279-301.

[4] Yamamoto K, Kawano H, Gando M, et al. Poor trunk flexibility is associated with arterial stiffening. Am J Physiol Heart Circ Physiol 2009;207:1314-8.

[5] Duanping DK, Arnett HA, Tyroler WA, et al. Arterial stiffness and the development of hypertension: The ARIC Study Hypertension 1999;34:201-6.

[6] Oliver JJ, Webb DJ. Noninvasive assessment of arterial stiffness and risk of atherosclerotic events. Arterioscler Thromb Vasc Biol 2003;23:554-66.

[7] Sutton-Tyrrell K, Najjar SS, Boudreau RM et al. Elevated aortic pulse wave velocity, a marker of arterial stiffness, predicts cardiovascular events in well-functioning older adults. Circulation 2005;111:3384-90.

[8] Hamilton PK, Lockhart CJ,Quinn CE, McVeigh GE.Arterial stiffness: clinical relevance, measurement, and treatment. Clinl Sci (London) 2007;113:157-70.

[9] Seals D.Habitual exercise and the age-associated decline in large artery compliance. Exerc Sport Sci Rev 2003;31:68-72.

[10] Vaitkevicius P, Fleg J, Engel J,et al. Effects of age and aerobic capacity on arterial stiffness in healthy adults. Circulation 1993; 88: 1456-62.

[11] Weber T, Auer J, O'Rouke MF, et al. Arterial stiffness, wave reflections, and the risk of coronary artery disease. Circulation 2004; 109:184-9.

[12] Cortez-Cooper MY, Anton MM, Devan AE, et al. The effects of strength training on central arterial compliance in middle-aged and older adults. Eur J Cardiovasc Prev Rehabil 2008;15:149-55.

[13] Kawano H, Tanaka H, Miyachi M. Resistance training and arterial compliance: keeping the benefits while minimizing the stiffening. $J$ Hypertens 2006;24:1753-9.

[14] Yoshizawa M, Maeda S, Miyaki A, et al. Effect of 12 weeks of moderate-intensity resistance training on arterial stiffness: a randomized controlled trial in women aged 32-59. Br J Sports Med 2009;43:615-8.

[15] Garber CE, Blissmer B, Deschenes MR, et al. American College of Sports Medicine. Position Stand: Quantity and quality of Exercise for developing and maintaining cardiorespiratory, musculoskeletal and neuromotor fitness in apparently healthy adults; guidelines for prescribing exercise. Med Sci Sports Exerc 2011;43:1334-59.

[16] Nelson ME, Rejeski WJ, Blair SN, et al. Physical activity and public health in older adults: recommendations from the american college of sports medicine and the american heart association. Med Sci Sports Exerc 2007;39:1435-45.

[17] Lu X,Hui-Chin CW, Tsang WW. Tai Chi, arterial compliance, and muscle strength in older adults. Eur J Cardiovasc Prev Cardiol Epup ahead of print 4 April 2012.

[18] Douris P, Chinan A, Gomez M, et al. Fitness levels of middle aged martial art practitioners Br J Sports Med 2004;38:143-7.

[19] Douris PC, Elokda AS, Handrakis JP, et al. Martial Art Training Enhances the Glutathione Antioxidant System in Middle Aged Adults. $J$ Strength Cond Res 2009;23:1518-21.

[20] Pickering TG, Hall JE, Appel LJ,et al. Recommendations for blood pressure measurement in humans and experimental animals: Part 1: blood pressure measurement in humans: a statement for professionals from the Subcommittee of Professional and Public Education of the American Heart Association Council on High Blood Pressure Research. Hypertension 2005;45:142-61.

[21] Hickson SS, Butlin M, Broad J, et al. Validity and repeatability of the Vicorder apparatus: a comparison with the SphygmoCor device. Hypertens Res 2009;32:1079-85.

[22] ACSM's Guidelines for Exercise Testing and Prescription. $8^{\text {th }}$ edition. Philadelphia: Lippincott Williams \& Wilkins, 2010; Pp:99-101.

[23] Baltaci G, Un N, Tunay V, et al. Comparison of three different sit and reach tests for measurement of hamstring flexibility in female university students. Br J Sports Med 2003;37:59-61.

[24] Field A. Discovering Statistics Using SPSS. ${ }^{\text {rd }}$ ed. Thousand Oak: Sage Publications. 2009; P: 373.

[25] Portney LG,Watkins MP. Foundations of Clinical Research. Applications to Practice. 3 rd ed. Upper Saddle River: Pearson Prentice Hall Health. 2009; Pp: 280, 480-9.

[26] Vlachopoulos C, Aznaouridis K, Stefanadis C. Prediction of cardiovascular events and all-cause mortality with arterial stiffness. $J$ Am Coll Cardiol 2010;55:1318-27.

[27] Mattace-Rasso FU, Hofman A, Verwoert GC, et al. Determinants of pulse wave velocity in healthy people and in the presence of cardiovascular risk factors: 'establishing normal and reference values'. Eur Heart J 2010;31:2338-50.

[28] Buckwalter, JA, Woo SL, Goldberg VM, et al. Soft-tissue aging and musculoskeletal function. J Bone Joint Surg Am 1993;75:153348.

[29] Boreham CA, Ferreira I, Twisk JW, et al. Cardiorespiratory fitness, physical fitness and arterial stiffness: the Northern Ireland Young Hearts Project. Hypertension 2004;44:721-6. 